\title{
Firmness Measurement of Stored 'Delicious' Apples by Sensory Methods, Magness-Taylor, and Sonic Transmission
}

\author{
Judith A. Abbott ${ }^{1}$ Henry A. Affeldt ${ }^{2}$, and Louis A. Liljedahl ${ }^{3}$ \\ Instrumentation and Sensing Laboratory, Beltsville Agricultural Research Center, Agricultural \\ Research Service, U.S. Department of Agriculture, Beltsville, MD 20705
}

Additional index words. Malus domestica, ripeness, quality, postharvest

\begin{abstract}
Delicious' apples (Malus domestica Borkh.) from five major U.S. production areas were tested after $\approx 3$ months of commercial storage. Soluble solids concentration (SSC), titratable acidity (TA), Magness-Taylor (MT) firmness, and sonic transmission spectra were compared with ripeness (maturity in trade terminology) scores assigned by six U.S. Dept. of Agriculture (USDA)-licensed apple inspectors according to USDA Grades and Standards inspection procedures. USDA ripeness categories are defined by textural and flavor terms. Inspectors in this test used visual, manual, oral, and auditory sensations to make their judgments, but firmness was the paramount characteristic judged. SSC and TA did not correlate with inspectors' scores, MT, or sonic measurements and thus are not satisfactory indices of ripeness for stored apples. Sonic resonance functions correlated significantly with mean inspectors' scores and with MT firmness. Inspectors' scores correlated slightly better with MT firmness than with sonic terms. MT is destructive and site-specific; in contrast, sonic measurements are nondestructive and representative of the entire fruit.
\end{abstract}

Apple production in the United States in 1987 was nearly 4.9 million tons, valued at more than $\$ 906$ million (U.S. Dept. Agr., 1989). Effective merchandising of such a volume of fruit requires effective sorting for several characteristics, particularly those in the Grade Standards (U.S. Dept. Agr., 1976). Domestic and foreign buyers both continuously demand better quality and a more uniform degree of ripeness.

Renewed interest in apple ripeness (maturity in trade terminology) by the Agricultural Marketing Service (AMS) caused us to reevaluate simple tests that AMS inspectors could use and to examine the characteristics and methods that inspectors presently use. It also afforded an opportunity to reexamine sonic vibrational characteristics for measuring texture in view of recent advances in electronic instruments and computers.

SSC and TA content of apples are known to change with maturation and ripening. Texture often is equated with quality in apples, with firm, crisp apples being the ideal. Firmness is also frequently used as a measure of maturity and ripeness. In fact, the USDA-AMS Standards for maturity/ripeness categories for fresh apples are Immature, Hard, Firm, Firm-ripe, Ripe, and Overripe (U.S. Dept. Agr., 1976). AMS inspectors rely mostly on firmness to make those assessments, particularly on apples that have been stored.

Firmness is most commonly measured by a puncture test using a MT type fruit firmness tester (Abbott et al., 1976; Blanpied et al., 1978; Bourne, 1974; Magness and Taylor, 1925). Provided that the probe geometry is comparable to that of the orig-

Received for publication 9 Sept. 1991. Accepted for publication 24 Feb. 1992 We wish to express our appreciation to the Agricultural Marketing Service (AMS) inspectors who participated in this study and to the staff of the Horticultural Crops Quality Laboratory, particularly the technicians who worked frantically to measure nearly 1000 apples in 3 days. Use of proprietary product names is" for identification only and does not imply endorsement of the product named or criticism of similar products not mentioned. The cost of publishing this paper was defrayed in part by the payment of page charges. Under postal regulations, this paper therefore must be hereby marked advertisement solely to indicate this fact.

${ }^{1}$ Research Horticulturist.

${ }^{2}$ Agricultural Engineer. Present address: Sunkist Growers, 760 E. Sunkist St., Ontario, CA 91761.

${ }^{3}$ Agricultural Engineer. Retired. inal MT, results from puncture tests are referred to generically as MT firmness (formerly, pressure). Puncture tests measure a small localized sample of tissue. The destructive nature of the puncture test obviously precludes its use for on-line sorting or extensive sampling. AMS inspectors use the MT firmness test to verify some of their judgments, but the reporting of MT values is not mandatory.

Nondestructive techniques for measuring firmness by use of vibrational characteristics have been proposed. Vibrational tests characterize the entire specimen, rather than being site-specific. Clark and Mikelson (1942) developed and patented a fruit ripeness tester based on the observation that the natural vibrational frequency of intact fruit changed during ripening. Falk et al. (1958) proposed the use of resonance frequency of potato ( $\mathrm{So}$ lanum tuberosum L.) tissue as a measure of its rigidity. Nybom (1962) estimated raspberry (Rubus idaeus L.) maturity by measuring the amount (amplitude) of sound transmitted through the berries. Abbott et al. (1968a, 1968b) reported that resonances in apples are influenced significantly by fruit maturity and ripeness, firmness, size, and cuts and bruises. Also, they proposed combining the apple's resonance frequency with its mass in the form $f^{2} m$ (termed a "stiffness coefficient") to estimate the dynamic elastic properties of the fruit flesh and to measure maturity/ripeness. They recommended using the lowest frequency flexural resonance, although other resonance frequencies also were related to ripeness and firmness. There have been numerous other attempts to use various aspects of the vibrational behavior of fruits and vegetables to detector to sort for texture or ripeness (Affeldt and Abbott, 1989; Armstrong, 1989; Armstrong et al., 1989; Bower and Rohrbach, 1976; Clark and Shackelford, 1973; Cooke, 1970, 1972; Finney, 1970, 1971; Finney et al., 1978, 1967, 1968; Garrett and Furry, 1972; Hamann and Carroll, 1971; Hamann et al., 1973; Peleg et al., 1990; Yamamoto et al., 1980, 1981). The development of the sonic measurements described in those references was a significant achievement, but the methods for large fruits have remained time-consuming, impractical for commercial application,

Abbreviations: MT, Magness-Taylor; SSC, soluble solids concentration, TA, titratable acidity. 
and limited in accuracy by the amount of information the data acquisition system could gather.

Our objective was to evaluate measurements that could improve fresh apple maturity/ripeness classification during USDA inspections. SSC, TA, MT firmness, and sonic transmission spectra were compared with ripeness scores assigned by USDAlicensed apple inspectors.

\section{Materials and Methods}

Apples. We examined commercial 'Delicious' apples of mixed, unspecified strains from the 1987 harvest. Apples were selected during USDA inspections in the first week of Jan. 1988, so they had been stored 3 to 4 months. Inspectors in Illinois, Michigan, New York, Virginia, and Washington each provided $\approx 200$ apples covering the broadest possible range of ripeness stages. Storage conditions were unspecified and included both normal refrigerated and controlled atmosphere storages. The apples were shipped by express mail to Beltsville, Md., usually taking 2 days.

The apples were held for up to 1 week at OC while all shipments were accumulated. Apples were equilibrated overnight at $22 \mathrm{C}$ in their boxes. They were numbered consecutively as removed from the boxes without regard for any indication of ripeness. Masses, diameters, and sonic spectra (described below) were recorded. Apples from each state were randomized separately and placed in trays of 20 to 23 apples to facilitate handling. The apples were returned to $\mathrm{OC}$ for 2 days before additional testing. Trays from all states henceforth were tested in random order.

Sonic measurements. The sonic resonance techniques were related to those of Finney and coworkers (Finney, 1970; Finney et al., 1978). Methods of vibrational excitement and data analysis differed in keeping with recent technological advances (Fig. 1). More than 50 combinations of contact media and support structures were tested in preliminary tests.

Intact apples were mounted horizontally on an electromagnetic vibrator (acoustical driver) (MB Dynamics, Cleveland, Model EA 1500; frequency range 5 to $10,000 \mathrm{~Hz}$; force vector). 225 N Floral clay (contact medium) was used to anchor an apple to a small aluminum pedestal attached to the vibrator (Affeldt and Abbott, 1989). A time-compressed electronic signal, equivalent to a 5- to $2000-\mathrm{Hz}$ sinusoidal scan, was fed to a power

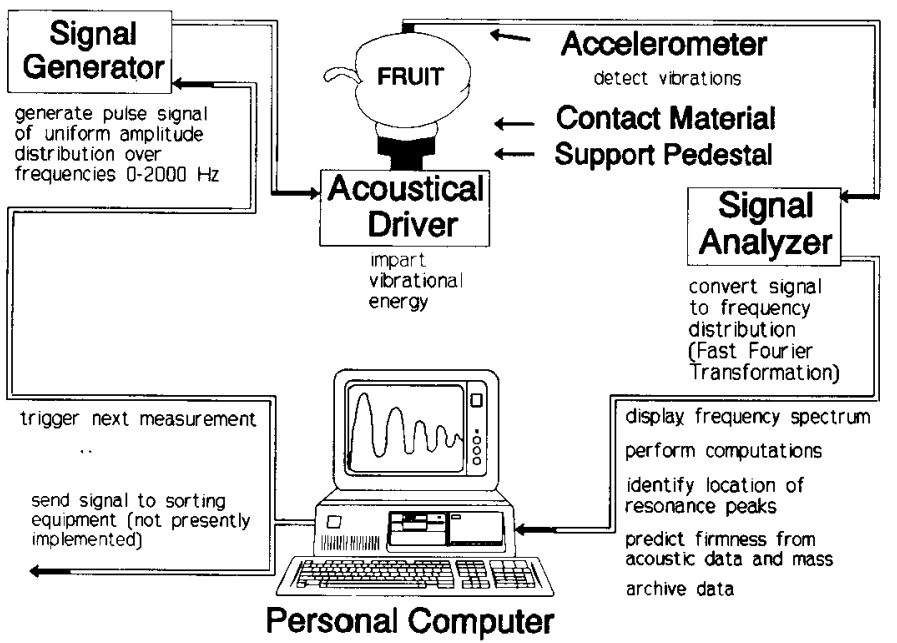

Fig. 1. Schematic diagram of sonic transmission measurement equipment (instruments identified in text). amplifier (signal generator) (Advanced Dynamic Instrumentation, Dallas, Model N100, frequency range 5 to $20,000 \mathrm{~Hz}$ ) to drive the vibrator's electromagnet. The resulting pulse caused input vibrations of constant acceleration from 5 to $2000 \mathrm{~Hz}$. An accelerometer (Endevco, San Juan Capistrano, Calif., Model 2222 , frequency response flat from 10 to $5000 \mathrm{~Hz} \pm 10 \%$, mass $=0.5 \mathrm{~g}$ ) was held firmly against the apple vertically opposite the vibrator. The vibrations of the apple were detected by the accelerometer. A signal analyzer (Hewlett Packard, Rockville, Md., 3561A Dynamic Signal Analyzer) collected signals in the time domain from the accelerometer, averaged the responses to 10 consecutive pulses at a single site, and converted the response to the frequency domain by fast fourier transformation. The frequency domain signal was then transferred to a computer. A complete response curve was obtained in $<1 \mathrm{sec}$ (Fig. 2 ).

A peak-detection program was written to extract the amplitudes and frequencies of the second and third resonances (peaks) and the valley separating them. We verified all computer-extracted values by visual checks of the curves. Erroneous data were corrected before further computations were performed. The stiffness coefficient (Abbott et al., 1968a, 1968b) was calcu-

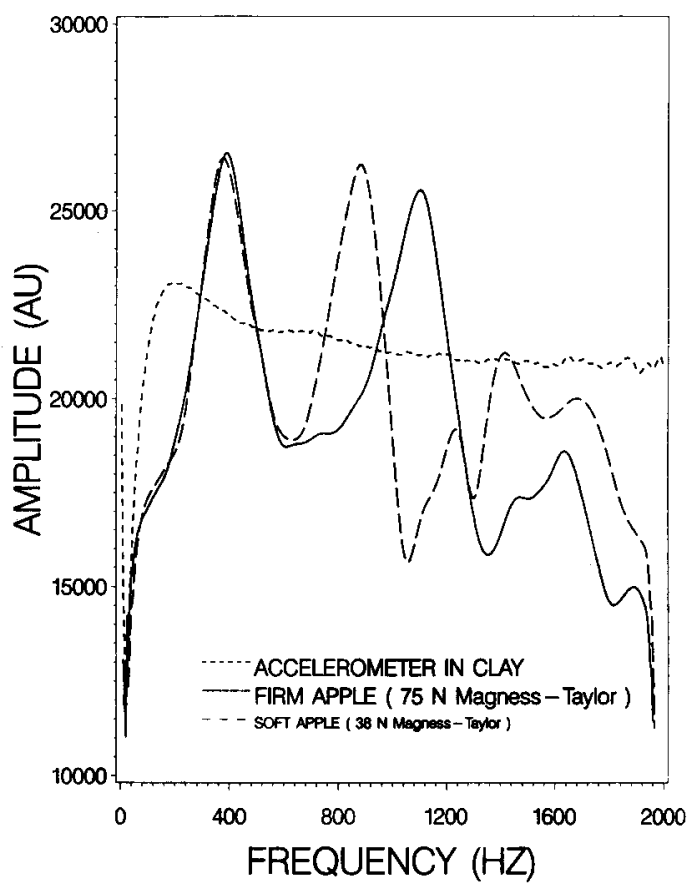

Fig. 2. Sonic excitation and transmission spectra. "Accelerometer in clay" represents excitation energy applied to apples. Remaining spectra are transmission for 'Delicious' apples of similar mass but different firmness.

Table 1. Correlation coefficients between mean ripeness scores and instrument measurements $(\mathrm{n}=702)$.

\begin{tabular}{lc}
\hline \hline Measurements & $\begin{array}{c}\text { Correlation } \\
\text { coefficients }\end{array}$ \\
\hline TA & 0.232 \\
SSC & 0.262 \\
MT & 0.834 \\
MT + TA & 0.834 \\
MT + SSC & 0.840 \\
MT + SSC/TA & 0.836 \\
MT + SSC + TA & 0.840 \\
\hline
\end{tabular}


Table 2. Correlation coefficients between each inspector's ripeness scores and the mean ripeness score of the other five inspectors or the designated instrument measurements $(n=702)$.

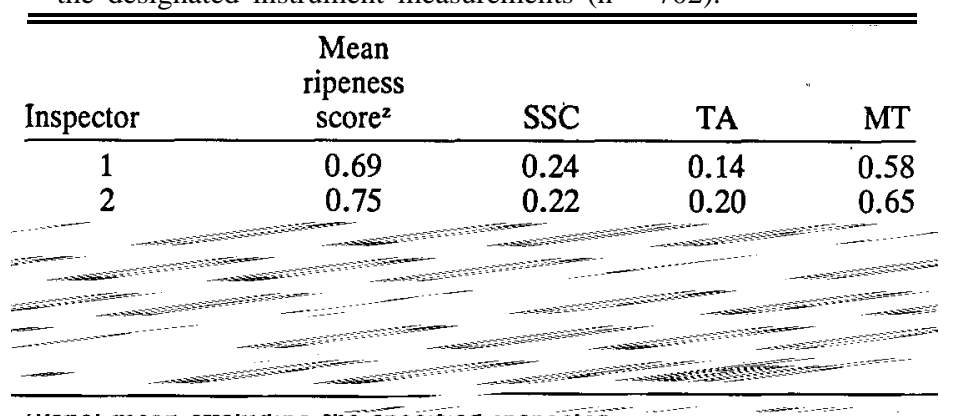

“Yanel mean excluaing tne speciried inspector.

lated for each apple as the product of its mass times the square of its second resonance frequency $\left(\mathrm{f}_{\mathrm{n}=2}\right)^{2} \mathrm{~m}$.

Firmness and SSC. Six supervisory-level USDA-licensed inspectors from apple-producing regions (Michigan, New York, Pennsylvania, Virginia, and Washington) and central distribution centers (Chicago) convened at our laboratory to evaluate the apples. A single inspector measured MT firmness manually on all apples within one tray, using the standard 11-mm apple probe and puncturing the brush and opposite sides. Another inspector collected on a hand refractometer the juice that exuded from the MT punctures and recorded the percent SSC for each apple. Measurement and inspection duties were rotated among the six inspectors on a one- or two-tray basis to limit bias, maximize efficiency, and minimize fatigue.

Ripeness assessment. Apples from one tray were arrayed, following MT/SSC measurement, on a wooden table under fluorescent lights for inspection. Ordinary office-type lighting was used because appearance was not to be evaluated and did not vary greatly. One inspector at a time assessed the ripeness of each apple from that tray, cycling through all six inspectors in random order. We instructed the inspectors to score maturity/ ripeness as defined in the AMS standards (U.S. Dept. Agr., 1976) and to disregard the other characteristics normally scored. They were given no instructions as to how to evaluate ripeness except to do it as they normally did during inspection.

Ripeness scores were converted to numerical values as they were entered into a computer by observers (not inspectors): 5 = Hard, 4 = Firm, 3 = Firm-ripe, 2 = Ripe, 1 = Overripe, and $0=$ severely overripe or too physiologically or mechanically damaged to evaluate. The ripeness scale is numbered according to increasing firmness. No apples were judged to be immature. AMS ripeness categories allow for time in marketing channels; thus, apples rated Hard are mature, but extremely firm, and apples rated Ripe, or perhaps even Overripe, might be considered to be of good eating quality at the time of measurement. Apples given a 0 by any inspector were later eliminated from the analyses.

The observers also noted the techniques used by the various inspectors.

$T A$. Following ripeness assessment, the flesh of each apple was sampled for TA. An amount equivalent to about one-fourth of the intact apple was juiced in a home-style electric fruit juicer (Acme Juicerator, Acme Juicer Mfg. Co., Waring Products Div., New Hartford, Corm.) with a strip of Whatman \#1 chromatography paper fitted inside the strainer to facilitate cleaning between apples. A 5-ml aliquot of juice was mixed with $20 \mathrm{ml}$ of water and five drops of hämatoxylin dye. The mixture was titrated with $0.01 \mathrm{~N} \mathrm{NaOH}$ to the point of distinct color change ( $\mathrm{pH}$ 7.0). Volume of $\mathrm{NaOH}$ was recorded and percent TA was calculated. We used the dye method instead of a $\mathrm{pH}$ meter because it is less expensive and easier for an inspector to perform at a remote inspection station. Titrations were performed by various technicians.

Statistical treatment. A completely randomized design with different numbers of apples from the five states was used. Grouping onto trays was ignored. Any apple from which any datum was missing was eliminated. Analyses are based on 702 apples.

MT data from both sides of each apple were averaged. Ripeness scores from the six inspectors were analyzed separately and also were averaged. Means are reported except where stated. The sonics test results are the values for a single site per apple, as compared with means of two sites for MT tests or six inspectors, putting sonics measurements at a statistical disadvantage.

Averaging over the six inspectors created 22 discrete mean ripeness scores. We pooled apples by mean ripeness scores to

Table 3. Correlation coefficients between sonic measurements and ripeness scores or MT firmness.

\begin{tabular}{|c|c|c|c|}
\hline Variable $^{\mathbf{z}}$ & Sonic variable & $\begin{array}{c}\text { Mean } \\
\text { ripeness }\end{array}$ & MT \\
\hline \multirow[t]{2}{*}{ Peak 1} & Amplitude & 0.16 & 0.23 \\
\hline & Frequency & 0.47 & 0.48 \\
\hline \multirow[t]{2}{*}{ Peak 2} & Amplitude & -0.07 & -0.13 \\
\hline & Frequency & 0.59 & 0.62 \\
\hline \multirow[t]{2}{*}{ Valley } & Amplitude & -0.36 & -0.40 \\
\hline & Frequency & 0.36 & 0.38 \\
\hline \multicolumn{2}{|l|}{ Amplitude at best fixed frequency } & -0.56 & -0.58 \\
\hline \multicolumn{2}{|l|}{ Mass } & -0.35 & 0.37 \\
\hline \multicolumn{2}{|l|}{ Stiffness coefficient ${ }^{y}$} & 0.60 & 0.64 \\
\hline \multirow[t]{2}{*}{ Multiple regression model ${ }^{\mathbf{x}}$} & $F_{n=1}+F_{n=2}$ & 0.59 & 0.62 \\
\hline & $F_{n-1}+F_{n-2}+$ Mass & 0.63 & 0.66 \\
\hline
\end{tabular}

"Peaks 1 and 2 and valley refer to each apple's individual resonance frequencies and amplitudes. "Amplitude at best fixed frequency" refers to the amplitude at the best single fixed frequency for the entire set of apples selected by a regression program testing the amplitudes at each frequency (in increments of $5 \mathrm{~Hz}$ ). The best frequencies selected were $835 \mathrm{~Hz}$ for ripeness and $840 \mathrm{~Hz}$ for MT. y Calculated as $\left(f_{n}-2\right)^{2} m$.

'The $R$ including frequencies of both peaks. 
Table 4. Correlation coefficients among peak 2 resonance frequency, stiffness coefficient, ripeness scores, and MT firmness calculated using means of 22 ripeness categories.

\begin{tabular}{|c|c|c|}
\hline Variable & $\begin{array}{l}\text { Ripeness } \\
\text { scores }\end{array}$ & MT \\
\hline MT firmness & 0.968 & -- \\
\hline Peak 2 frequency & 0.981 & 0.968 \\
\hline Stiffness coefficient ${ }^{2}$ & 0.974 & 0.983 \\
\hline
\end{tabular}

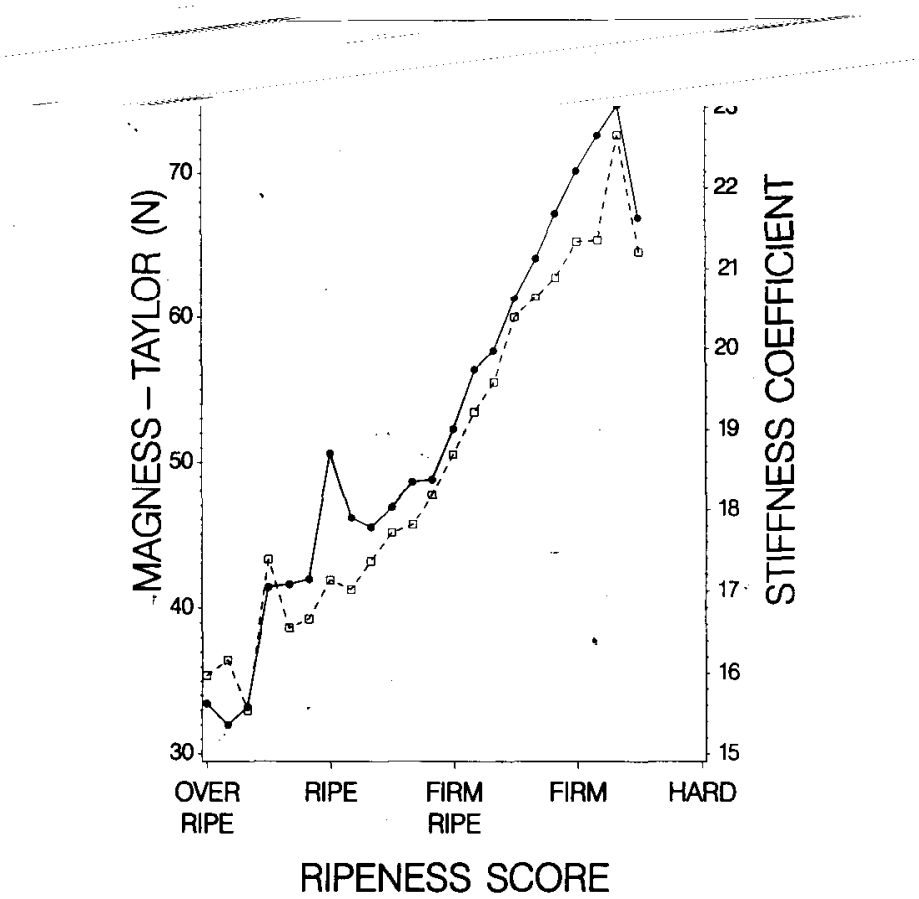

Fig. 3. MT firmness and sonic stiffness coefficient $\left[\left(\mathrm{f}_{\mathrm{n}-2}\right)^{2} \mathrm{~m} \times\right.$ $\left.10^{-8}\right]$ vs. ripeness scores. Each point represents the mean of one to 60 apples with the same mean ripeness score. Solid line $=$ stiffness coefficient; broken line $=$ MT firmness.

create 22 ripeness categories with 1 to 60 apples in any category. Correlations among MT, ripeness scores, and sonics also were computed using the category means.

The "amplitude at the best fixed frequency" (see Table 3) was found by performing regressions of ripeness scores or MT on sonic transmission amplitudes at each frequency (in increments of $5 \mathrm{~Hz}$ ) and selecting the highest regression coefficient.

\section{Results and Discussion}

Inspectors were observed to use visual inspection of the external and internal surfaces, hand pressure, thumb pressure, cutting (perception of resistance and sound), biting, and chewing to reach their judgments. When asked what they tasted for, each inspector had a slightly different response. All stressed textural terms, primarily crispness, toughness, and mealiness. Some added that they consider flavor, sweetness, and sourness slightly and that starchiness and greenness are important near harvest. The inspectors appeared to integrate visual, manual, oral, and auditory sensations to evaluate ripeness, but firmness was the paramount characteristic.

Each inspector needed to cut a slice from each apple for tasting, so they may have taken smaller slices than normal. Because of damage to the apple by successive inspectors, the condition of the apples (particularly bruising) changed during inspection. The order of inspectors was random among lots of apples, so changing condition should not have been a biasing factor. However, changing condition probably contributed to variance, particularly for apples that fell near the margin between two ripeness categories.

AMS maturity/ripeness is evaluated primarily in terms of texture, though ripeness and flavor terms appear in the definitions along with textural terms. This fact was confirmed by regressing the inspectors' ripeness scores on SSC, TA, and MT. Correlation coefficients were very low for SSC and TA, whether using the panel mean (Table 1) or individual inspectors' scores (Table 2). Combining SSC and TA with MT to predict ripeness scores using stepwise multiple regression analysis (Table 1), we found no significant contribution from SSC or TA. We conclude that, at least for 'Delicious' apples stored 3 or 4 months, sweetness and acidity as estimated by SSC and TA were not major considerations in judging AMS ripeness and, therefore, could not provide an objective method for measuring either AMS ripeness or firmness.

Ripeness scores varied among inspectors, and correlation coefficients were higher for mean scores than for individuals' scores (Table 2), as expected. Contributions of SSC, TA, and MT to ripeness scores differed slightly among inspectors (Table 2 ). Because of these differences, mean scores were used in regression analysis with instrument measurements. Selecting only the "best" or the three best inspectors for comparisons with MT or sonics did not alter the conclusions; therefore, only the six-inspector means are reported.

Sonic spectra of apples presented in the past generally were based on scanning successive frequencies and were time-consuming to measure. We compared scanned and time-compressed (pulse) spectra for individual apples. They differed slightly, especially at the higher frequencies. However, the frequencies of the first and second resonances were comparable between scanned and pulsed spectra, particularly the second resonance, which previously had been related to changes in apple texture (Abbott et al., 1968a, 1968b).

Clay has been used as the contact medium in several previous studies (Finney, 1970, 1971; Finney et al., 1978). However, clay is time-consuming to apply and tends to adhere to the fruit. More than 50 combinations of coupling materials and support structures were tested to find a suitable substitute (Affeldt and Abbott, 1989). The material from which the support form was made and its shape significantly altered the transmission spectra. An aluminum or plexiglass pedestal, a shallow conical aluminum form, or a concave wooden hemispherical form minimized amplitude attenuation and shifts in detected resonance frequencies. Floral clay provided the best contact; other types of clay and plasticize were not satisfactory. Alternatively, a ring of floral clay sealed in latex surgical glove material provided nearly the same efficiency as the exposed clay. This configuration cradled the apple, and the latex resisted slippage without adhering. Some other materials also provided satisfactory coupling, although transmission of the impulse was not as good as with floral clay. Frequency shifts were minimized when the coupling medium was a fine granular material, but amplitudes were generally lower than with floral clay. Commercially gleaned sand, 60/80 micron glass beads (Wilkens Instrument and Research, Walnut Creek, Calif.), and confectioners' sugar (Domino) best transmitted vibrations that induced well-defined resonance peaks. Dry, fine, granular materials will conform to the contours of individual apples when excited with a high-energy $3-$ to $5-\mathrm{kHz}$ 
sonic burst. A loose covering of thin plastic film, such as commercial food wrap, could be used to contain the granular materials and prevent contamination of the apples without alteration of the spectra.

Floral clay in a shallow conical aluminum form provided the best transmission of vibrational energy to the apple and was used for all data reported here. Amplitudes of vibration transmitted through the supporting clay (Fig. 2) are almost equal throughout the spectrum. Exaggerated amplitudes at specific frequencies on the apple spectra result from the response of the apples' structure to periodic stimulation; peaks correspond to "resonances."

A few apples were measured at $45^{\circ}$ increments around the equator (Affeldt and Abbott, 1989). Frequencies of the second resonance peaks shifted less than $15 \mathrm{~Hz}$ for reasonably symmetrical apples. Variations in flexural harmonics were evident beyond $\mathrm{f}_{\mathrm{n}=2}$. Measurements were repeatable within $\pm 5 \mathrm{~Hz}$ at a particular site. Large bruises, gross asymmetry, and other irregularities caused distortions in the spectra.

Conspicuous features of the sonic spectra of apples are the amplitudes and frequencies of the first and second peaks and of the valley (minimum) separating them. We correlated these singly and in combinations with the ripeness scores and with MT firmness. Correlations between ripeness or MT and sonic resonance amplitudes were never significantly higher than correlations with resonance frequency and were often much lower (Table 3). Amplitudes depend on positioning and the integrity of apple-to-vibrator and apple-to-detector contacts, as well as the apple characteristics. Resonance frequencies primarily depend on the mechanical properties of the apple. Abbott et al. (1968a, 1968b) identified the frequency of the second peak as being the spectral feature most sensitive to apple texture. Finney and coworkers (1970-78) also used that value. Present results reconfirm the choice.

In previous studies, the mass was found to correlate highly negatively with resonance frequencies, and we again found that to be true. To compensate for the effect of apple size on resonance frequencies, Abbott et al. (1968b) proposed a stiffness coefficient based on the formula for Young's modulus of elasticity, the square of the second resonance frequency times the mass, written $\left(f_{n=2}\right)^{2} m$ and abbreviated henceforth $f^{2} m$. We tested the frequencies, the stiffness coefficient, and a simple additive model combining frequency and mass against the inspectors' ripeness scores.

The sonic resonance frequency is closely related to the ripeness scores and MT firmness, although the correlation coefficients are not particularly high $(r \leq 0.62)$ (Table 3$)$. Between MT values for opposite sides of each apple, $r=0.89$. The best correlation we can realistically expect to achieve between MT firmness and any other measurement will be of similar magnitude. When all the apples with a given mean ripeness score were averaged and ripeness categories were compared, the correlations among sonic resonance frequencies, MT, and ripeness scores were very high $(r>0.96, \mathrm{n}=22)$ (Table 4, Fig. 3). The apparent disparity between the two hardest classes in Fig. 3 is probably an artifact due to the very few apples in those ripeness classes $(n=2$ and $n=1)$.

Different mechanical properties are measured by MT puncture and sonic resonance tests. The properties measured by humans making destructive cutting or biting tests are intuitively and fundamentally more closely related to the properties measured by the destructive MT puncture test (mainly strength) than to those tested at the miniscule deformations involved in the nondestructive sonic resonance test (mainly elasticity). However, sonic resonance provides an estimate of the firmness of apples that relates reasonably well to destructive measurements.

To the extent that firmness is the dominant attribute of the AMS ripeness score, sonic resonance may provide a rapid, nondestructive measurement that could be used for fresh-apple ripeness classification. The sonic resonance technique also permits repeated measurement of the same individual fruit over time for following physiological changes.

\section{Literature Cited}

Abbott, J.A., G.S. Bachman, N.F. Childers, J.V. Fitzgerald, and F.J. Matusik. 1968a. Sonic techniques for measuring texture of fruits and vegetables. Food Technol. 22(5):101-112.

Abbott, J.A., N.F. Childers, G.S. Bachman, J.V. Fitzgerald, and F.J. Matusik. 1968b. Acoustic vibration for detecting textural quality of apples. Proc. Amer. Soc. Hort. Sci. 93:725-737.

Abbott, J.A., A.E. Watada, and D.R. Massie. 1976. Effe-gi, MagnessTaylor, and Instron fruit pressure testing devices for apples, peaches, and nectarines. J. Amer. Soc. Hort. Sci. 101:698-700.

Affeldt, H.A. and J.A. Abbott. 1989. Apple firmness and sensory quality using contact acoustic transmission. Proc. 7th Intl. Congr. on Agr. Eng., Dublin, Ireland, vol. 3:2037-2045.

Armstrong, P.R. 1989. Measurement of apple firmness using the acoustic impulse response. PhD Diss., Michigan State Univ., East Lansing.

Armstrong, P.R., H.R. Zapp, and G.K. Brown. 1989. Impulse excitation of acoustic vibrations in apples for firmness determination. Amer. Soc. Agr. Eng. Paper no. 89-3052.

Blanpied, G.D., W.J. Bramlage, D.H. Dewey, R.L. LaBelle, L.M. Massey, Jr., G.E. Mattus, W.C. Stiles, and A.E. Watada. 1978. A standardized method for collecting apple pressure test data. New York Food and Life Sci. Bul. 74.

Bourne, M.C. 1974. Comparison of results from the use of the Magness-Taylor pressure tip in hand- and machine-operation. J. Texture Studies 5:105-108.

Bower, D.R. and R.P. Rohrbach. 1976. Application of vibrational sorting to blueberry firmness separation. Trans. Amer. Soc. Agr. Eng. 19:185-191.

Clark, H.L. and W. Mikelson. 1942. Fruit ripeness tester. U.S. Patent 2277037.

Clark, R.L. and P.S. Shackelford. 1973. Resonance and optical properties of peaches as related to flesh firmness. Trans. Amer. Soc. Agr. Eng. 16:1140-1142.

Cooke, J.R. 1970. A theoretical analysis of the resonance of intact apples. Amer. Soc. Agr. Eng. Paper no. 70-345.

Cooke, J.R. 1972. An interpretation of the resonant behavior of intact fruits and vegetables. Trans. Amer. Soc. Agr. Eng. 15(6):10751080.

Falk, S., C.H. Hertz, and H.I. Virgin. 1958. On the relation between turgor pressure and tissue rigidity. Physiol. Plant. 11:802-817.

Finney, E.E. 1970. Mechanical resonance within Red Delicious apples and its relation to fruit texture. Trans. Amer. Soc. Agr. Eng. 13:177180.

Finney, E.E. 1971. Dynamic elastic properties and sensory quality of apple fruit. J. Texture Studies 2:62-74.

Finney, E.E., J.A. Abbott, A.E. Watada, and D.R. Massie. 1978. Nondestructive sonic resonance and the texture of apples. J. Amer. Soc. Hort. Sci. 103:158-162.

Finney, E.E., I. Ben-gara, and D.R. Massie. 1967. An objective evaluation of changes in firmness of ripening bananas using a sonic technique. J. Food Sci. 32:642-646.

Finney, E.E. and K.H. Norris. 1968. Instrumentation for investigating dynamic mechanical properties of fruits and vegetables. Trans. Amer. Soc. Agr. Eng. 11(1):94-97.

Garrett, R.E. and R.B. Furry. 1972. Velocity of sonic pulses in apples. Trans. Amer. Soc. Agr. Eng. 15(4):770-774.

Hamann, D.D. and D.E. Carroll. 1971. Ripeness sorting of Muscadine 
grapes by use of low-frequency vibrational energy. J. Food Sci. 36:1049-1051.

Hamann, D.D., L.J. Kushman, and W.E. Ballinger. 1973. Sorting blueberries for quality by vibration. J. Amer. Soc. Hort. Sci. 98:572-576

Magness, J.R. and G.F. Taylor. 1925. Unimproved type of pressure tester for the detemination of fruit maturity. U.S. Dept. Agr. Circ. 350, p. 8.

Nybom, N. 1962. A new principle for measuring firmness of fruits. Hort. Res. 2:1-8.

Peleg, K., U. Ben-Hanan, and S. Hinga. 1990. Classification of avocado by firmness and maturity. J. Texture Studies 21:123-139.
U.S. Dept. of Agriculture. 1976. United States standards for grades of apples. Agr. Mktg. Serv., Washington, D.C.

U.S. Dept. of Agriculture. 1989. Agricultural statistics 1989. U.S. Govt. Printing Office, Washington, D.C.

Yamamoto, H., M. Iwamoto, and S. Haginuma. 1980. Acoustic impulse response method for measuring natural frequency of intact fruits and preliminary applications to internal quality evaluation of apples and watermelons. J. Texture Studies 11:117-136.

Yamamoto, H., M. Iwamoto, and S. Haginuma. 1981. Nondestructive acoustic impulse response method for measuring internal quality of apples and watermelons. J. Jpn. Soc. Hort. Sci. 50(2):247-261. 